\section{Case Reports in Acute Medicine}

Case Rep Acute Med 2020;3:79-82

This article is licensed under the Creative Commons Attribution-NonCommercial 4.0 International License (CC BY-NC) (http://www.karger.com/Services/OpenAccessLicense). Usage and distribution for commercial purposes requires written permission.

\title{
Acute Myocardial Infarction Complicated by Very Severe Hypertriglyceridemia
}

\author{
Gregory Wu Brianna Bockman Jesse Perez \\ Ross University School of Medicine, Miramar, FL, USA
}

\section{Keywords}

Hypertriglyceridemia · Myocardial infarction · Pancreatitis · Emergency medicine $\cdot$ Critical care

\begin{abstract}
Hypertriglyceridemia (HTG) is the elevation of serum triglyceride levels above $150 \mathrm{mg} / \mathrm{dL}$ and is linked to complications such as pancreatitis and increased mortality risk. An uncommonly known co-presentation of HTG is acute ST-elevation myocardial infarction (STEMI), with the literature citing only one other case of acute STEMI complicated by very severe HTG. We report on a 36-year-old male with multiple comorbidities presenting with acute STEMI and elevated pancreatic enzymes suggestive of pancreatitis. A subsequent lipid profile revealed very severe HTG with a triglyceride level of 2,700 $\mathrm{mg} / \mathrm{dL}$. We discuss the pathogenesis, management, and screening guidelines of very severe HTG. Clinicians should be aware that acute STEMI complicated by HTG is rare and that earlier detection and intervention may allow for the prevention of life-threatening complications.

(C) 2020 The Author(s)

Published by S. Karger AG, Basel
\end{abstract}

\section{Introduction}

Hypertriglyceridemia (HTG) is defined as the elevation of fasting serum triglyceride above $150 \mathrm{mg} / \mathrm{dL}$ and can be categorized into mild, moderate, severe, and very severe [1]. HTG has known associations with cardiovascular complications and increased risk of mortality [2]. Elevated triglyceride levels may lead to symptoms such as eruptive xanthomas, lipemia 


\section{Case Reports in Acute Medicine}

Wu et al.: Acute Myocardial Infarction Complicated by Very Severe HTG

retinalis, and lipemic plasma. Severe HTG (SHTG) and very severe HTG (VSHTG) may lead to the well-studied acute pancreatitis and fatty liver disease. A less understood and extremely rare complication of HTG may be acute ST-elevation myocardial infarction (STEMI).

We present the case of an acute STEMI as well as elevated pancreatic enzymes suggestive of pancreatitis in a young male with VSHTG.

\section{Case Report}

A 36-year-old Hispanic male with a past medical history of hypertension, diabetes, obesity, and alcohol and cocaine use not on any active medications presented to the emergency department with 2 days of pressure-like left-sided chest pain associated with nausea and vomiting. Of note, the patient denied any smoking history and denied any known family history, including of lipid disorders. The patient was severely obese with a BMI of 39.7; otherwise, his physical examination was unremarkable without physical findings of HTG such as eruptive xanthomas or lipemia retinalis.

Collected laboratory results revealed a troponin level of $0.292 \mathrm{ng} / \mathrm{mL}$, ethanol level of 415 $\mathrm{mg} / \mathrm{dL}$, lipase of $171 \mathrm{U} / \mathrm{L}$, blood glucose of $140 \mathrm{mmol} / \mathrm{L}, \mathrm{HgA}_{1 \mathrm{c}}$ of $6.5 \%$, and electrocardiography findings of ST-elevation within the anterolateral leads. The patient received aspirin and a nitroglycerin drip and was taken for emergent cardiac catheterization. An $80 \%$ mid-left anterior descending artery lesion was noted and a non-drug-eluting stent was placed, accompanied by initiation of clopidogrel.

Subsequent laboratory results revealed a triglyceride level of $2,700 \mathrm{mg} / \mathrm{dL}$. The patient was started on an insulin drip with downtrending triglyceride levels to $551 \mathrm{mg} / \mathrm{dL}$ on HOD\#4, with blood glucose within the range of 99-120 mmol/L daily. He was discharged on HOD\#5, based on a triglyceride level below $500 \mathrm{mg} / \mathrm{dL}$ and an improved clinical status. He received proper counseling and appropriate cardiac and triglyceride-reducing medications upon discharge.

\section{Discussion and Conclusion}

HTG is defined as fasting serum triglyceride levels typically greater than $150 \mathrm{mg} / \mathrm{dL}$ [1]. Elevation of triglyceride levels has been associated with increased risk for cardiovascular events and overall mortality [2]. Risk factors for HTG include metabolic abnormalities such as obesity, excessive alcohol intake, diabetes, and genetic disorders. SHTG is characterized by levels above $1,000 \mathrm{mg} / \mathrm{dL}$, while VSHTG is above $2,000 \mathrm{mg} / \mathrm{dL}$, both carrying the additional risk of pancreatitis [1]. Other symptoms of SHTG may include xanthomas, dyspnea, hepatosplenomegaly, abdominal pain, and impaired cognition. A 2018 retrospective analysis determined the prevalence of VSHTG among hospitalized patients $(>2,000 \mathrm{mg} / \mathrm{dL})$ to be $0.01 \%$ [3]. An extensive review of the literature revealed only one report of SHTG complicating acute myocardial infarction [4]. Further, there are no reports of VSHTG presenting in a patient with both acute myocardial infarction and elevated lipase levels suggestive of pancreatitis simultaneously.

SHTG results from the overproduction of very low-density lipoprotein by the liver and overproduction of chylomicrons by the intestine. Patients with SHTG tend to have mono- or polygenic disorders in addition to typical secondary factors such as the aforementioned metabolic abnormalities. One such disorder is monogenic chylomicronemia, a rare condition in 


\section{Case Reports in Acute Medicine}

Wu et al.: Acute Myocardial Infarction Complicated by Very Severe HTG

which affected individuals have a severe deficiency in lipoprotein lipase and apolipoprotein C-II, resulting in a significant reduction of lipolysis of triglycerides [5]. Some forms of the polygenic disorder, type $\mathrm{V}$ hyperlipoproteinemia, result in severe elevation of triglycerides due to an increased very low-density lipoprotein concentration and plasma chylomicron concentration, with involvement of the ligand apoE4 possibly implicated [6]. While SHTG is not independently implicated in atherosclerotic cardiovascular disease events including myocardial infarction, the hyperviscosity resulting from severely elevated triglycerides can contribute to endothelial dysfunction and tissue ischemia [7]. Furthermore, individuals with SHTG have been found to have significantly elevated plasma fibrinogen and factor Xc; elevated fibrinogen is a risk factor for cardiovascular mortality, while elevated factor Xc is found in patients at high risk for ischemic heart disease [8].

For asymptomatic patients, HTG can be managed with low-fat diets, fibrates, statins, and omega-3 fatty acids. However, patients with VSHTG usually require aggressive treatment that is dependent on the symptoms and etiology. Patients with HTG-associated acute pancreatitis or HTG with concurrent hyperglycemia should be admitted to the hospital with insulin therapy. Refractory HTG, SHTG-induced pancreatitis, and hyperviscosity symptoms may be managed with plasmapheresis, with a study showing an $80 \%$ reduction in plasma triglycerides after initiation [9]. Hospitalized patients should not be discharged until triglyceride levels fall below $500 \mathrm{mg} / \mathrm{dL}$ or the patient is clinically stable [10].

Screening guidelines for detection of dyslipidemia are debated in the literature as the mortality benefit of early detection has yet to be proven. Current guidelines advise lipid screening before puberty (9-11 years) and after puberty (17-21 years) [11]. In adults, it is recommended to screen earlier to evaluate the cardiovascular risk, appropriately stratify the risk, and initiate lipid-lowering agents. A large retrospective study revealed that among patients presenting with triglycerides above $2,000 \mathrm{mg} / \mathrm{dL}, 43 \%$ were not taking any triglyceridereducing medication [12]. Further findings suggest that in follow-up appointments for patients with VSHTG, health care providers addressed HTG in only $50 \%$ of the patients, with triglyceride re-measurements in only $18 \%$ of the patients [12]. These reports highlight the necessity for an increased consistency in screening and surveillance of patients with an elevated lipid profile that places them at risk for adverse events.

Clinicians should be aware of rarely occurring acute STEMIs complicated by VSHTG. Although our patient had many other risk factors such as Hispanic ethnicity, uncontrolled diabetes, obesity, and alcohol and cocaine use, we believe that his VSHTG may have been a poorly understood cause of the acute STEMI. Preventative strategies including earlier screening, frequent surveillance, and prompt intervention with triglyceride-lowering agents may reduce the risk of acute adverse events and improve outcomes in patients with VSHTG. Further efforts should be focused on establishing the relationship between SHTG/VSHTG and acute STEMI in order to develop an understanding of acute management and prognosis.

\section{Statement of Ethics}

The authors have no ethical conflicts to disclose. Informed consent was obtained from the patient for the publication of this case report. There are no patient identifiers in the case report which may link the patient to the report. 


\section{Case Reports in Acute Medicine}

\section{Conflict of Interest Statement}

The authors have no conflicts of interest to declare.

\section{Funding Sources}

The authors did not receive any financial support.

\section{Author Contributions}

All authors drafted the manuscript, and all have read, reviewed, and approved the final transcript.

\section{References}

1 Berglund L, Brunzell JD, Goldberg AC, Goldberg IJ, Sacks F, Murad MH, et al.; Endocrine society. Evaluation and treatment of hypertriglyceridemia: an Endocrine Society clinical practice guideline. J Clin Endocrinol Metab. 2012 Sep;97(9):2969-89.

2 Tenenbaum A, Klempfner R, Fisman EZ. Hypertriglyceridemia: a too long unfairly neglected major cardiovascular risk factor. Cardiovasc Diabetol. 2014 Dec;13(1):159.

3 Chyzhyk V, Kozmic S, Brown AS, Hudgins LC, Starc TJ, Davila AD, et al. Extreme hypertriglyceridemia: genetic diversity, pancreatitis, pregnancy, and prevalence. J Clin Lipidol. 2019 Jan - Feb;13(1):89-99.

4 Divakaran A, Sedhom R, Patel V, Khan W. Severe Hypertriglyceridemia Complicating ST Elevation Myocardial Infarction. In: A57. Critical Care Case Reports: Cardiovascular Disease II. American Thoracic Society International Conference Abstracts. American Thoracic Society; 2017:A1963-A1963. doi:

5 Fojo SS, Brewer HB. Hypertriglyceridaemia due to genetic defects in lipoprotein lipase and apolipoprotein CII. J Intern Med. 1992 Jun;231(6):669-77.

6 Ghiselli G, Schaefer EJ, Zech LA, Gregg RE, Brewer HB Jr. Increased prevalence of apolipoprotein E4 in type V hyperlipoproteinemia. J Clin Invest. 1982 Aug;70(2):474-7.

7 Rosenson RS, Shott S, Lu L, Tangney CC. Hypertriglyceridemia and other factors associated with plasma viscosity. Am J Med. 2001 Apr;110(6):488-92.

8 Simpson HC, Mann JI, Meade TW, Chakrabarti R, Stirling Y, Woolf L. Hypertriglyceridaemia and hypercoagulability. Lancet. 1983 Apr;1(8328):786-90.

9 Gubenšek J, Buturović-Ponikvar J, Marn-Pernat A, Kovac J, Knap B, Premru V, et al. Treatment of hyperlipidemic acute pancreatitis with plasma exchange: a single-center experience. Ther Apher Dial. 2009 Aug;13(4):314-7.

10 Jialal I, Amess W, Kaur M. Management of hypertriglyceridemia in the diabetic patient. Curr Diab Rep. 2010 Aug;10(4):316-20.

11 Expert Panel on Integrated Guidelines for Cardiovascular Health and Risk Reduction in Children and AdolescentsNational Heart, Lung, and Blood Institute. Expert panel on integrated guidelines for cardiovascular health and risk reduction in children and adolescents: summary report. Pediatrics. 2011 Dec;128 Suppl 5:S213-56.

12 Esparza MI, Li X, Adams-Huet B, Vasandani C, Vora A, Das SR, et al. Very Severe Hypertriglyceridemia in a Large US County Health Care System: Associated Conditions and Management. J Endocr Soc. 2019 May;3(8):1595-607. 